\title{
Dysphagia with Schatzki Ring or Steakhouse Syndrome: Better to Scope than to Watch
}

\author{
Kyawzaw Lin ${ }^{1 *}$, Purav Patel ${ }^{1}$, Liauw MXW'2, Pwint Phyu Hlaing ${ }^{3}$ and Linus Yoe ${ }^{4}$ \\ ${ }^{1}$ Internal Medicine Department, The Brooklyn Hospital Center, Affiliate of the Mount Sinai Hospital. 121, Dekalb Avenue, \\ Brooklyn, NY 11201, USA \\ ${ }^{2}$ Medical Student, St. George University, West Indies \\ ${ }^{3}$ University of Medicine (I), Yangon, Myanmar
}

${ }^{4}$ Internal Medicine Department, Associate Program Director, IM Residency and Clerkship Director, The Brooklyn Hospital Center, Affiliate of the Mount Sinai Hospital. 121, Dekalb Avenue, Brooklyn, NY 11201, USA

Received: May 10, 2018; Accepted: June 06, 2018; Published: June 11, 2018

*Corresponding author: Kyawzaw Lin, Department of Medicine, The Brooklyn Hospital Center, 1733 West 1st Street, Brooklyn, NY 11223, USA, Tel: 315664-1916; E-mail: dr.kyawzawlin2015@gmail.com

\begin{abstract}
A Schatzki ring is a thin, smooth ring causing constriction at the esophagogastric junction. We present the case of an 82-year-old man with six months of episodic dysphagia with a Schatzki ring. The patient, who is a chronic heavy smoker, was admitted to the hospital due to episodic dysphagia and an unexplained 30-pound weight loss. A review of his history and a physical examination were unremarkable. The dysphagia did not follow a specific type of solid food intake. Laboratory results were within normal reference ranges except thyroid hormone panel. An esophagogram showed mild esophageal dysmotility with mildly delayed clearance. Esophagogastroduodenoscopy showed a Schatzki ring located in the distal third of the esophagus with mild gastritis and arteriovenous malformation in the duodenal bulb. Histopathology showed gastroesophageal reflux disease and no squamous epithelial dysplasia or intestinal metaplasia. The patient improved clinically and tolerated a normal diet after two to three days on methimazole and proton pump inhibitors.

The diameter of the lumen is the main factor in determining the presence of dysphagia. It is the most common cause of chronic episodic dysphagia in adults. However, in our patient, the diameter of SR is not significantly small enough to cause dysphagia during endoscopy. Dysphagia responded well to conservative management after initiation of methimazole for Hashimato's thyroiditis and proton pump inhibitor.
\end{abstract}

\section{Introduction}

A Schatzki ring (SR) is a thin symmetric circumferential constriction composed of mucosa and submucosa at the gastroesophageal junction. It was first described by Schatzki and Gary in 1953 [1]. The incidence was approximately 6\% to $14 \%$. SR is the most common cause of chronic episodic dysphagia in adults $[1,2]$. Here, we present the case of an 82-year-old healthy man with six months of episodic dysphagia with an SR.

\section{Case Presentation}

An 82-year-old man who is a chronic heavy smoker was admitted to the hospital for evaluation after a six-month period of episodic dysphagia accompanied by an unexplained 30-pound weight loss. His dysphagia was not precipitated by a specific type of solid food intake such as meat or bread. The patient denied any associated cardiopulmonary, abdominal, or constitutional symptoms. The physical examination was unremarkable. His 30-year smoking history was considered pertinent. Laboratory results were within reference ranges with the exception of thyroid markers: his serum thyroid stimulating hormone level was 0 $\mathrm{ng} / \mathrm{mL}$, free T4 was $2.5 \mathrm{ng} / \mathrm{mL}$, free T3 was $8.0 \mathrm{ng} / \mathrm{mL}$, serum thyroglobulin antibody level was $53.10 \mathrm{IU} / \mathrm{mL}$, thyroid peroxidase $\mathrm{Ab}$ was $57.90 \mathrm{IU} / \mathrm{mL}$ and his thyroid stimulating immunoglobulin level was $6.57 \mathrm{IU} / \mathrm{mL}$. A thyroid scan found thyroid uptake in the low normal range at $13.5 \%$ and $12.6 \%$ (reference range: $10 \%$ to $30 \%$ ), giving the impression of thyroiditis. A thyroid sonogram indicated a diffusely enlarged hypervascular heterogeneous

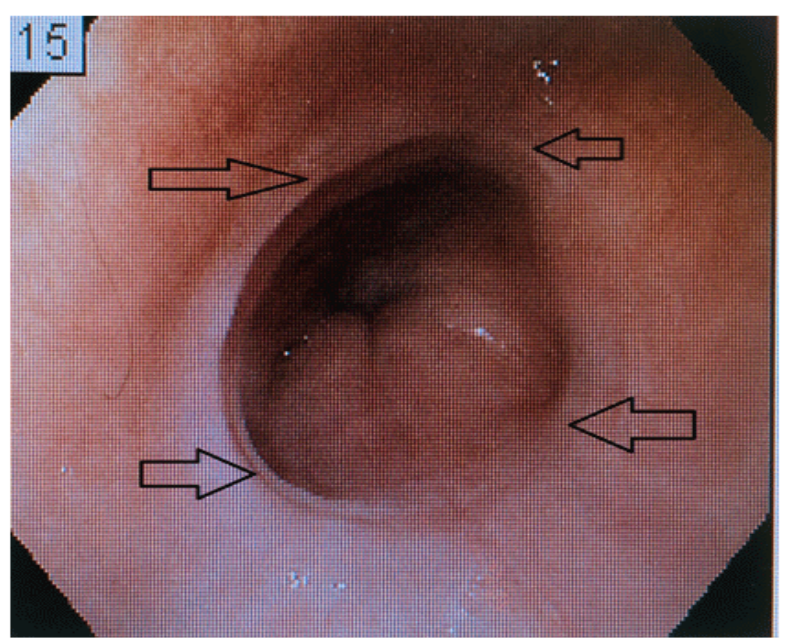

Figure 1: A Schatzki ring located in the distal third of the esophagus (black arrows) 


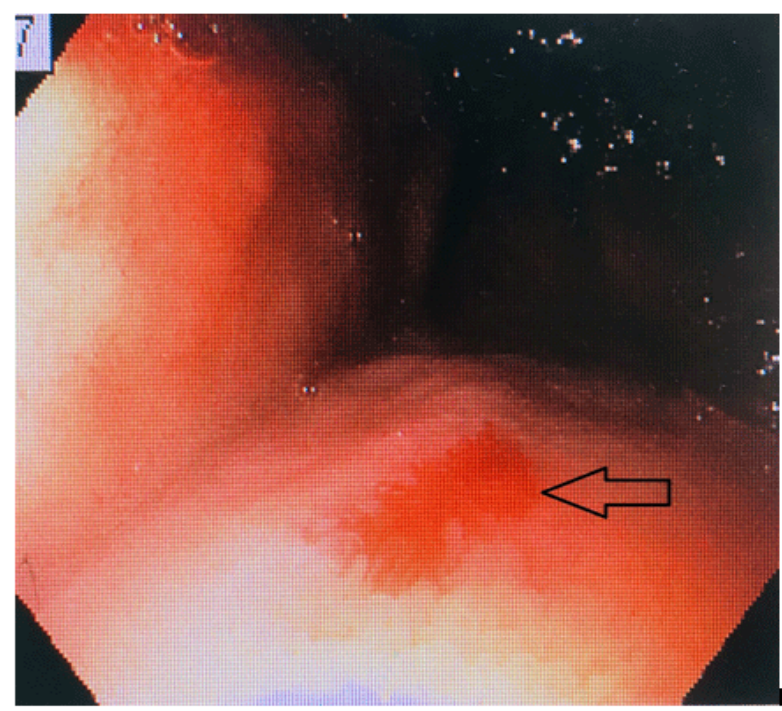

Figure 2: Arteriovenous malformation in the duodenal bulb (black arrow)

thyroid gland consistent with thyroiditis. An esophagogram showed mild esophageal dysmotility with mildly delayed clearance. There was no evidence of stricture, obstruction, mass lesion or infectious/inflammatory changes in the esophagus. A computed tomography (CT) scan with contrast of the soft tissue of the neck showed an air-filled dilatation of the thoracic esophagus. Esophagogastroduodenoscopy showed an SR located in the distal third of the esophagus with mild gastritis (Figure 1) and an arteriovenous malformation in the duodenal bulb (Figure 2). Histopathology results showed evidence of gastroesophageal reflux disease but no squamous epithelial dysplasia or intestinal metaplasia. The patient improved clinically and tolerated a normal diet after a few days of treatment with proton pump inhibitors and anti-thyroid medication for Hashimoto's thyroiditis.

\section{Discussion}

Generally, an esophageal ring is defined as a thin, smooth, and constricting ring of normal esophageal tissue composed of three layers: mucosa, submucosa, and muscular fibers. However, in the SR, there is only mucosa and submucosa [3]. Microscopically, the upper surface of the SR is covered by squamous epithelium and the lower surface by columnar epithelium. Both Schatzki and Gary [1] and Ingelfinger and Kramer [4] were pioneers in correlating episodic dysphagia with the SR. Ingelfinger and Kramer [4] postulated that the SR is a constriction due to an active motor phenomenon in the setting of intermittent contraction and bunching up of the musculature. However, Schatzki and Gary [1] reported that the SR was formed by passive means. The exact mechanism of SR formation is still unknown. SRs are rarely found in children and are mostly found in adults over the age of 40 in the West [5].

Most SRs are asymptomatic and have a good prognosis and outcome. Dysphagia due to an SR is usually intermittent, episodic, and non-progressive to solid food intake such as steak or bread and occurs during hurried ingestion or under stressful conditions. Rarely do they present with acute food bolus impaction as symptoms typically resolve with the passage of the food bolus. Perforation and pneumatosis of the thorax or mediastinum is one of the rare but fatal complications.

The first step in the diagnosis is to obtain the pertinent medical history. A barium esophagogram is commonly used to explore esophageal lesions such as webs or rings. In a barium esophagogram, SRs are usually seen as a thin circumferential membrane projecting into the esophageal lumen at a right angle to the long axis of the lower part of the structure. Often, they mimic lower esophageal malignancy with the presence of irregular filling defects caused by inflammatory scarring from associated acid reflux and shouldering. Thus, histologic tissue samples and diagnostic endoscopy are needed to rule out malignancy. However, there is no reported correlation between SRs and esophageal cancers [5].

Conservative methods are usually applied for asymptomatic patients. They are advised to eat smaller, more thoroughly chewed food bolus and not to eat too quickly. Treatment is reserved for symptomatic groups when dietary measures have failed. Treatments include dilatation and rupture of the ring or excision of the ring with repair of a hiatal hernia. Large-bore endoscopic dilatation or bougienage is the mainstay of therapy. In Ottinger et al. [6], 36 patients with SRs treated by rupture of the ring and subsequent repair were followed up for six years. Of those, $37 \%$ were symptom-free, $23 \%$ presented with persistent mild symptoms and $40 \%$ had a recurrence. However, dilatation is contraindicated in eosinophilic esophagitis, Bullous disease, Zenker diverticulum, and, in some cases, has been shown to have a high risk of perforation. Even post-dilatation, judicious use of acid-suppressing therapy is reported to be superior to placebo in reduction of recurrences [7].

In our case, symptomatic dysphagia work-up for failure to thrive in a 82-year old chronic smoker had been done. During the EGD, Schatzki's ring was found but the diameter of SR is not significantly narrow enough to create symptomatic dysphagia. During the work up, severe hyperthyroidism had been detected in blood tests. Dysphagia responded well to conservative management after initiation of methimazole for Hashimato's thyroiditis.

\section{Conclusion}

The diameter of the lumen is the main factor in the determination of the presence of dysphagia. However, in our patient, the luminal diameter of SR is not significantly narrow during endoscopy. Dysphagia responded well to conservative management after initiation of methimazole for Hashimato's thyroiditis and proton pump inhibitor. In summary, symptomatic dysphagia resulting in failure to thrive might be due to underlying hyperthyroidism from Hashimoto's thyroiditis in this 82 -year old chronic smoker. 


\section{Author Acknowledgement}

Contributors: All the participant authors contributed equally and approved the submitted manuscript.

Patient consent: Obtained.

Author Contribution Obtained: Dr Kyawzaw Lin contributed equally in gathering information, writing introduction, case report and discussion. Dr.Purav Patel, Michael Liauw( Medical Student), Dr. Pwint Phyu Hlaing contributed in Editing. Dr. Linus Yoe provided the optimal patient care and management of the case.

\section{References}

1. Schatzki R, Gary JE. Dysphagia due to a diaphragm-like localized narrowing in the lower esophagus (lower esophageal ring). Am J Roentgenol Radium Ther Nucl Med. 1953;70(6):911-922.

2. Krishnamurthy C, Hilden K, Peterson KA, Mattek N, Adler DG, FangJC. Endoscopic findings in patients presenting with dysphagia: analysis of a national endoscopy database. Dysphagia. 2012;27(1):101-105. doi:10.1007/s00455-011-9346-0
3. Ott DJ, Ledbetter MS, Chen MY, Koufman JA, Gelfand DW. Correlation of lower esophageal mucosal ring and 24-h pH monitoring of the esophagus. Am J Gastroenterol. 1996;91(1):61-64.

4. Ingelfinger FJ, Kramer P. Dysphagia produced by a contractile ring in the lower esophagus. Gastroenterology. 1953;23(3):419-430. doi: 10.1016/S0016-5085(53)80183-6

5. Marshall JB, Kretschmar JM, Diaz-Arias AA. Gastroesophageal reflux as a pathogenic factor in the development of symptomatic lower esophageal rings. Arch Intern Med. 1990;150(8):1669-1672. doi 10.1001/archinte.1990.00040031669014

6. Ottinger LW, WilkinsEWJ.Late resultsin patients with Schatzkirings undergoing destruction of the ring and hiatus herniorrhaphy. Am J Surg. 1980;139(4):591-594. doi:10.1016/0002-9610(80)90343-8

7. Sgouros SN, Vlachogiannakos J, Karamanolis G, Vassiliadis K, Stefanidis G, Bergele C, et al. Long-term acid suppressive therapy may prevent the relapse of lower esophageal (Schatzki's) rings: a prospective, randomized, placebo-controlled study. Am J Gastroenterol. 2005;100(9):1929-1934. doi: 10.1111/j.15720241.2005.41184.x 